\title{
Melting of two-dimensional colloidal crystals: A simulation study of the Yukawa system
}

\author{
Kevin J. Naidoo and Jurgen Schnitker \\ Department of Chemistry, University of Michigan, Ann Arbor, Michigan 48109-1055
}

(Received 13 July 1993; accepted 3 November 1993)

\begin{abstract}
The two-dimensional melting transition of charged polystyrene spheres in aqueous colloidal suspension has been studied by molecular dynamics simulation of a screened Coulomb system. Some central predictions of the Kosterlitz-Thouless-Halperin-Nelson-Young theory of defect-mediated melting are confirmed, such as an apparent divergence of the correlation lengths for translational and bond-orientational order at different thermodynamic state points, but there are also predictions of the theory that are violated. The defect topology is very complex, with oscillation periods of the defect density of many million time steps duration. The need for extensive sampling and, to a lesser degree, the choice of potential function continue to be the crucial issues for any attempt to generate a hexatic structure by means of computer simulation.
\end{abstract}

\section{INTRODUCTION}

Monodisperse colloidal particles in aqueous suspension provide a model system of unique versatility for the study of classical condensed phase phenomena. ${ }^{1,2}$ Thus such suspensions have recently been used in several kinds of experimental setups to study the nature of the melting transition in two-dimensionally (2D) restricted geometries. ${ }^{3-5}$ Surprisingly, the conclusions drawn from the various experiments are conflicting, adding to a multitude of contradictory data that have been obtained over the last decade and a half in numerous other investigations of 2D melting, both experimental and theoretical. ${ }^{6,7}$ Motivated by these unresolved controversies, we here present a computer simulation study of the 2D Yukawa system as a simple model for charged colloidal suspensions. The rationale is that simulation results will not only complement the experimental information, but may also help to clarify the origin and the essence of the ongoing disputes regarding the interpretation of the experiments.

The strong interest that has been shown in $2 \mathrm{D}$ melting for more than a decade emanates from the theory of defectmediated melting by Kosterlitz, Thouless, Halperin, Nelson, and Young (KTHNY). This famous theory predicts a peculiar two-step melting process. First, a continuous transition leads from the solid to a bond-orientationally ordered phase, a so-called "hexatic"; then a second transition, also continuous, leads from the hexatic phase to the liquid. ${ }^{8,9}$ This is to be opposed to the conventional view of melting, for systems in both $3 \mathrm{D}$ and $2 \mathrm{D}$, where a single first-order transition takes the system from the long-range (or quasi-long-range) ordered state of the solid phase to the disordered state of the liquid phase. The physical driving force behind the KTHNY transitions are longwavelength fluctuations that lead to the unbinding of defect pairs, specifically pairs of dislocations (solid $\rightarrow$ hexatic) and pairs of disclinations (hexatic $\rightarrow$ liquid). No account is made of short-wavelength fluctuations, related to effects of local packing and such phenomena as the possible occurrence of grain boundaries.

Because of the just mentioned incompleteness of
KTHNY theory, no consensus has emerged concerning its applicability to real systems. Nevertheless, it seems that at least some experimental systems, such as certain smectic liquid crystals, ${ }^{10}$ have provided good indications in support of the theory. Probably the most compelling evidence for KTHNY-type melting has been obtained by Murray et al. in experiments with colloidal suspensions of simple polystyrene spheres that are confined between glass plates. ${ }^{3}$ There are also other experimental systems with more indirect indications for two-step melting as prescribed by KTHNY theory. ${ }^{6}$

Even the experimental evidence for 2D colloidal suspensions is not uncontested ${ }^{4,5}$ (see Ref. 11 for a recent comprehensive review), reminiscent of the highly controversial interpretations that have been given of many of the numerous computer simulation studies of $2 \mathrm{D}$ melting. A good example of the latter predicament is provided by two recent papers on the hard-disk system that contain quite different inferences about the nature of the melting transition. ${ }^{12,13}$ Intricate issues also arise in the analysis of simulations of the inverse-twelfth-power-law system that we reexamined recently. ${ }^{14}$

Nevertheless, it appears that a majority of simulation results has been interpreted as indicative of a first-order transition, or at most a weak first-order transition, ${ }^{6,15}$ whereas at least some experimental data appear to provide definite hints in favor of KTHNY-type melting. It is not clear if this apparent disparity between experiment and simulation has to be attributed to (1) either fundamental differences between the types of systems that have been studied, or (2) inherent problems with the setup of current simulations, in particular regarding system size and degree of sampling, or (3) different methods for data analysis and interpretation.

Even though it can be argued that all of these issues are potentially relevant, the true significance of each point still needs to be determined. What appears to be called for is a systematic investigation of the same type of system with both experimental and simulation methods. This is the goal of the present paper. We conduct a series of very long molecular dynamics simulations of a crude model for 
charged colloidal suspensions, employing a simple, pairwise additive model potential. We then look for evidence similar to that of the experimental information. ${ }^{3-5}$ In particular we try to determine-just as in the experimental case-if structures from the intermediate region of the phase diagram exhibit all the features of a true hexatic or if they have to be attributed to conventional two-phase coexistence.

While several modest simulation studies of the (unscreened) 2D Coulomb system have been reported, ${ }^{16-19}$ there is only one previous simulation study of the 2D Yukawa system $^{20}$ and this study was mostly limited to a characterization of its thermodynamics. This is in stark contrast to the 3D Yukawa system which has received a lot of attention ${ }^{21-26}$ and whose melting transition has repeatedly been addressed from the viewpoint of phenomenological theories of melting. ${ }^{24-26}$ In this paper, we will ignore the 2D analogs of these theories and only focus on arguments for and against KTHNY-type melting, as obtained from a direct analysis of the microscopic structure. We also do not study the entire phase diagram, but rather work with one specific set of potential parameters-basically chosen ad hoc-such that the simulated system resembles in some ways the experimental colloidal sphere systems ${ }^{3-5}$ and in particular the systems of Murray et al. $^{3}$

The organization of this paper is as follows. In Sec. II, we describe the computational methods. The results are presented and discussed in Sec. III, first addressing the orientational and translational correlation functions, and then examining the defect density and defect topology. The final Sec. IV contains the conclusions.

\section{METHODS}

A system of 1225 particles in a periodically replicatêd rectangular unit cell of approximate area $A=(25 \times 42)$ $\mu \mathrm{m}^{2}$ was studied, corresponding to a $25 \times 49$ supercell of an unstrained triangular lattice. This is a fairly small system, but given the fact that size dependencies have to some extent been studied before, ${ }^{27,28}$ we decided to use the available computational resources for performing very long simulation runs. The simulations are performed in the traditional, strictly 2D simulation setup. (Based on experimental evidence concerning a dependency of the phase transition behavior on the dimensionality of the system, ${ }^{3(d), 29}$ one would expect-if anything-that this could imply a slight bias towards KTHNY-type melting behavior.)

The colloidal particles, suspended in a medium of dielectric constant $\varepsilon_{r}$, interact with the pairwise additive, Yukawa-type potential function

$$
U(r)=\frac{1}{\varepsilon_{r}} \frac{\left(z^{*}\right)^{2}}{r} e^{-\kappa r} .
$$

The charge $Z^{*}$ is an effective, renormalized charge that is much smaller than the actual charge $Z$. In Murray et al.'s experiments two different kinds of colloidal spheres were used whose renormalized charges have been estimated to be $Z^{*}=750 e_{0}$ and $Z^{*}=1000 e_{0} .^{3(\mathrm{~d})}$ We use $Z^{*}=750 e_{0}$ and the dielectric constant of water $\left(\varepsilon_{r}=78\right)$ in all simulations. For the Debye screening length $1 / \kappa$ we generally use $1 / \kappa=0.20 \mu \mathrm{m}$. It will later be seen that this yields a melting density about halfway between the melting densities of the Murray et al. two colloids. Some data were also collected for a screening length of $1 / \kappa=0.15 \mu \mathrm{m}$; no qualitatively different observations were made and results of these simulations will not be presented.

Of course the potential function (1) can only be expected to give a very crude and qualitative representation of the actual interactions in the experimental system under study. ${ }^{2,21(b), 23,30}$ For example, the potential is purely repulsive without a Hamaker-type attractive term, and we do not have an explicit geometric factor that could account for finite sphere size effects. These omissions should not be of concern for simulations in a fairly narrow phase transition region where we can simply take the charge $Z^{*}$ as a renormalized and practically density-independent value that represents all of the more subtle effects in an empirical and summary fashion. In general, one would expect the sphere-sphere interactions in a realistic model for the experimental 2D system to be even more complex than in the 3D bulk system, due to the image charges induces in the confining walls. We do not attempt to account for such effects.

The pair interactions are evaluated up to a cutoff distance of $r_{c}=2.3 \mu \mathrm{m}$. Since our simulations are in a sense loosely modeled on the experimental studies of Murray et $a l^{3}$ we use a particle mass of $8.95 \times 10^{9} \mathrm{amu}$. For an approximate density of $1.0 \mathrm{~g} / \mathrm{cm}^{3}$, this would correspond to spheres of $0.305 \mu \mathrm{m}$ diam, i.e., exactly the sphere size of both monodisperse colloids that have been studied experimentally. ${ }^{3}$

The equations of motion are integrated with the leapfrog Verlet algorithm. ${ }^{31}$ A roughly linear increase of the energy fluctuation with increasing time step shows a clear change in slope for time steps $>0.45 \mu \mathrm{s}$; therefore a time step of $\Delta t=0.35 \mu$ s (about $1 / 40$ of the Einstein vibrational period) is used. Runs of at least 130000 and sometimes 2 million steps are carried out. Certain runs are carried on even longer, for as long as 8 million or 22 million steps. The latter is equivalent to about $8 \mathrm{~s}$ of laboratory time.

NVT simulations are carried out, mostly using an isokinetic constraint algorithm. ${ }^{31}$ In the very long runs the system is alternatively coupled to a heat bath by periodic reinitialization of the velocities, ${ }^{31}$ thus presumably improving the sampling efficiency even more. The runs are carried out at room temperature $\left(20^{\circ} \mathrm{C}\right)$ and the phase transition is explored via density variation, similar to the experimental setups. ${ }^{3-5}$

To characterize the translational order, we calculate the total pair correlation function

$$
g(r)=\left\langle\delta\left(\mathbf{r}^{\prime}\right) \delta\left(\mathbf{r}^{\prime}-\mathbf{r}\right)\right\rangle,
$$

where the ensemble brackets are understood to imply proper normalization and averaging over all pairs of particles and all angles. At solid densitiès, we can also calcu- 
late the individual translational correlation functions that are obtained from the Fourier components $\rho_{G}$ of the microscopic density $\rho(r),{ }^{9}$

$$
g_{G}(r)=\left\langle\rho_{\mathbf{G}}^{*}\left(\mathbf{r}^{\prime}\right) \rho_{\mathbf{G}}\left(\mathbf{r}^{\prime}-\mathbf{r}\right)\right\rangle .
$$

Here $\rho_{\mathbf{G}}$ is the translational order parameter with respect to a particular reciprocal lattice vector $\mathbf{G}$ of the underlying crystal structure

$$
\rho_{\mathrm{G}}=e^{i \mathrm{G} \cdot \mathrm{r}} \text {. }
$$

We only consider the first three vectors of the triangular lattice, $\left|\mathbf{G}_{0}\right|=G_{0},\left|\mathbf{G}_{1}\right|=\sqrt{ } 3 G_{0}$, and $\left|\mathbf{G}_{2}\right|=2 G_{0}$, where $G_{0}$ $=4 \pi /\left(\sqrt{ } 3 a_{0}\right)$, with $a_{0}$ being the lattice spacing. The correlation function ( 3 ) is always averaged over the three equivalent crystallographic directions; if a particular configuration was obtained in a liquid-solid traverse (with possible "misorientation" relative to the computational unit cell) the exact lattice orientation is determined first.

The orientational order is characterized by calculating the bond-orientational correlation function $g_{6}(r)$,

$$
g_{6}(r)=\left\langle\Psi_{6}^{*}\left(\mathbf{r}^{\prime}\right) \Psi_{6}\left(\mathbf{r}^{\prime}-\mathbf{r}\right)\right\rangle,
$$

where $\Psi_{6}$ is the order parameter for the local orientation of the "grain" that is given by the "geometric bonds" (with angles $\phi_{k}$ relative to an arbitrary reference axis) of a given particle with its six closet neighbors $k$,

$$
\Psi_{6}=\frac{1}{6} \sum_{k=1}^{6} e^{6 i \varphi_{k}}
$$

As a measure of the deviation from triangular order, $\Psi_{6}$ has an absolute value of one for a particle in a perfectly ordered environment and represents the spatial orientation of the local "grain" through the phase factor. All correlation functions are calculated using fast-Fourier-transform (FFT) techniques, ${ }^{31}$ descretizing the relevant data on a $1024 \times 1024$ square lattice, ${ }^{14}$ and usually averaging the correlation functions over a number of independent configurations.

Finally, the defect structure is determined by Voronoi tessellation with a standard algorithm, ${ }^{31}$ identifying disclinations as particles with either more or less than six nearest neighbors.

\section{RESULTS AND DISCUSSION}

It is well-known ${ }^{32}$ that there is no volume change on melting in a $D$-dimensional system that interacts via an inverse-power-law potential $r^{-n}$ provided that $n<D$. From the equation of state, $P=f(\rho)$, of our simulated 2D system (not shown), we conclude that to a good approximation this apparently also applies to the smoothly truncated Yukawa potential. Thus there are virtually no constraints regarding the width of the potentially hexatic region.

The equation of state also indicates that the phase transition takes place at an approximate density of $\rho \approx 1.17$ $\mu \mathrm{m}^{-2}$; corresponding to a nearest neighbor distance of $a_{0}$ $=0.99 \mu \mathrm{m}$. In the experiments of Murray et al., freezing is observed at nearest neighbor distances of $a_{0}=0.78 \mu \mathrm{m}$
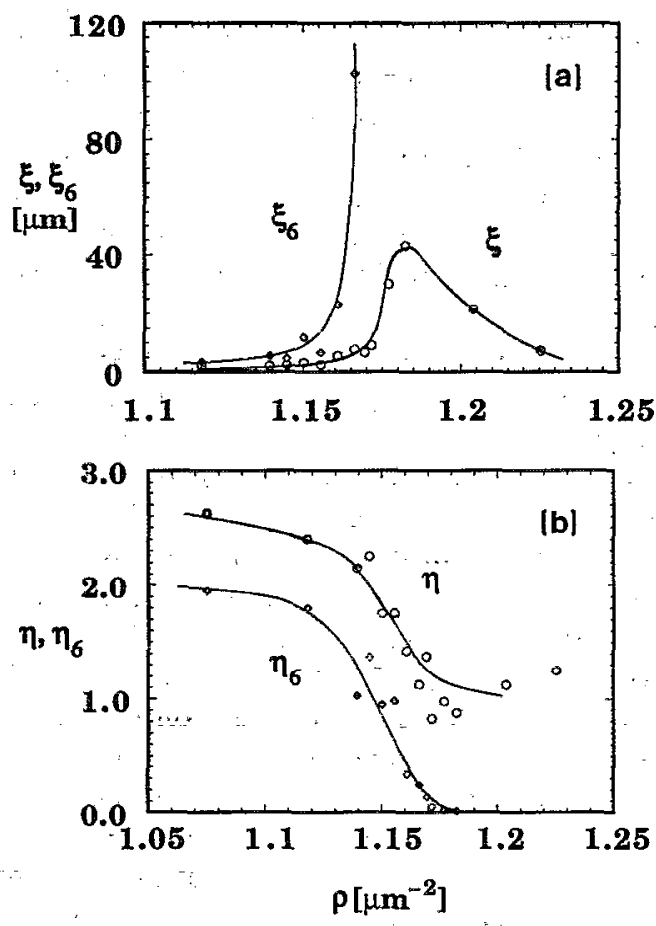

FIG. 1. (a) Correlation lengths $\xi$ and $\xi_{6}$ for assumed exponential decay of translational and orientational correlation functions $g(r)$ and $g_{6}(r)$, respectively. The lines drawn are only guides for the eye. (b) Power law exponents $\eta$ and $\eta_{6}$ for assumed algebraic decay of the same two correlation functions. The lines drawn are only guides for the eye.

[Ref. 3(a)] and $a_{0}=1.31 \mu \mathrm{m}$ [Ref. 3(d)], for their two choices of colloid. Our system thus lies about halfway between these two values.

\section{A. Orientational and translational order}

Figure 1(a) gives the correlation lengths $\xi_{6}$ and $\xi$ for orientational and translational order, respectively, as obtained by fitting the bond-orientational correlation function $g_{6}(r)$ and the pair correlation function $g(r)$ to exponential decay envelopes. In the two-step freezing scenario of KTHNY theory these correlation lengths diverge at different thermodynamic state points. If we analyze our data the same way as Murray and Van Winkle ${ }^{3(a)}$ and identify the onset of orientational and translational order with correlation lengths of $\xi_{6} \approx 30 a_{0}$ and $\xi \approx 15 a_{0}$, respectively, then we have to conclude that there are two distinct transitions at densities of $\rho \approx 1.161$ and $1.172 \mu \mathrm{m}^{-2}$, respectively. Thus there is an intermediate (and possibly hexatic) region that is about $\approx 1 \%$ wide in the density. An observation of this kind is at the heart of Murray and Van Winkle's original claim to have successfully identified a hexatic phase in the colloidal sphere system. ${ }^{3(a)}$

The correlation length for translational order in Fig. 1 (a) only grows to a maximum of $\xi \approx 45 a_{0}$, due to the fact that the order of a 2D solid is only quasi-long-ranged (actually giving rise to an algebraic decay law, see below), and probably also indicating a certain microgranularity of the simulated solid. This is unsatisfactory, but also seen in the experimental case. Thus for the Murray et al. two colloids 


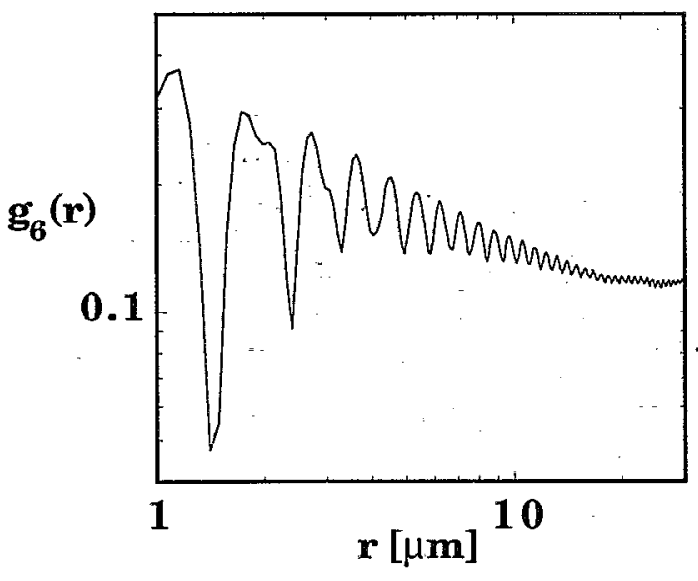

FIG. 2. Log- $\log$ plot of the orientational correlation function $g_{6}(r)$ at density $\rho=1.164 \mu \mathrm{m}^{-2}$.

a maximum occurs at $\xi \approx 90 a_{0}$ [Ref. $\left.3(\mathrm{a})\right]$ and $\xi \approx 40 a_{0}$ [Ref. 3(d)], and in the system of Tang et al. the translational correlation length does not even rise to more than $\xi \approx 10 a_{0}$ until far into the solid region. ${ }^{5}$ Hence our simulated system would in this regard appear to be not much worse than is the case under the best experimental conditions.

Figure $1(\mathrm{~b})$ is obtained by alternatively fitting $g_{6}(r)$ and $g(r)$ to algebraic decay envelopes $r^{-\eta / 6}$ and $r^{-\eta}$, respectively. The interpretation of the new figure is somewhat less clear; a particular impediment is the fact that the translational decay exponent $\eta$ barely falls below $\eta \approx 1.0$, again because of the only quasi-long-range positional order and the microgranularity of the solid. Nevertheless, the picture is at least consistent with a delay between the densities where orientational and translational order diverge.

A power-law decay is in fact expected for the bondorientational order in the presumed hexatic phase. Figure 2 gives a $\log -\log$ plot of the decay of the correlation function $g_{6}(r)$ at a density of $\rho=1.164 \mu \mathrm{m}^{-2}$, towards the liquid side of the intermediate region. Obviously, the correlation function can be well described by an algebraic decay law, at least up to a distance of $\approx 20 \mu \mathrm{m}$. (The deviations seen for larger distances would be consistent with the presence of a finite fraction of a solidlike phase.) More specifically, KTHNY theory predicts that the decay exponent drops continuously from a value of $\eta_{6}=1 / 4$ at the liquid to hexatic transition to $\eta_{6}=0$ at the hexatic to solid transition. ${ }^{9}$ From Fig. $1(\mathrm{~b})$, we find $\eta_{6} \approx 0.35$ at the density of $\rho \approx 1.161 \mu \mathrm{m}^{-2}$ that corresponds to the former of the two transitions. The predicted exponent of $\eta_{6}=1 / 4$ is not too far from this value and probably just outside the error bar of the simulation value.

We now turn to a more detailed analysis of the translational order. Figure 3 shows its decay upon reaching the melting density of $\rho=1.172 \mu \mathrm{m}^{-2}$, but now resolved with respect to the first few Fourier components of the microscopic density. Just as for the overall pair correlation function, the quasi-long-range order of the solid phase is expected to give rise to algebraic decays $r^{-\eta_{G}}$ of the

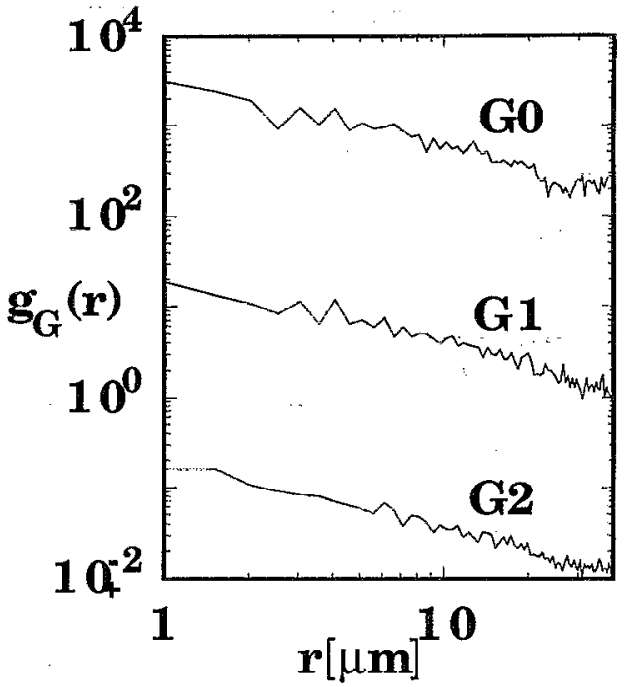

FIG. 3. $\log -\log$ plots of translational correlation functions $g_{G}(r)$ for first three reciprocal lattice vectors $\mathbf{G}_{0}, \mathbf{G}_{1}$, and $\mathbf{G}_{2}$, and at density $\rho=1.172$ $\mu \mathrm{m}^{-2}$. The exponent of the algebraic decay law, $r^{-\eta}$, is $\eta_{G} \approx 0.7$ in all three cases.

individual correlation functions $g_{G}(r)$. More specifically, KTHNY theory predicts that the decay exponents $\eta_{G}$ depend quadratically on the length of the Bragg vector $\mathbf{G}$ if the system is at the solid to hexatic transition. ${ }^{9}$ If $\lambda$ and $\mu$ denote the Lamé elastic constants we have

$$
\eta_{\mathbf{G}}(\text { solid-hexatic })=k T \frac{3 \mu+\lambda}{4 \pi \mu(2 \mu+\lambda)}|\mathbf{G}|^{2}
$$

Specifically for the first Bragg point, $\mathbf{G}_{0}$, the decay exponent at the transition is predicted ${ }^{9}$ to fall in the range $\eta_{0}=1 / 4-1 / 3$.

From the double-logarithmic representation of Fig. 3 it can be seen that the decay at a density on the borderline between solid and intermediate phase is in fact algebraic over the entire distance range $(r \leqslant 40 \mu \mathrm{m})$ for each of the first three reciprocal lattice vectors. However, there is hardly any difference between the three cases, and the deduced decay exponents $\eta_{0}, \eta_{1}$, and $\eta_{2}$ are all on the order of $\approx 0.7$, contrary to the prediction of the theory. Of course, our determination of the melting density may be quite inaccurate. We therefore present in Fig. 4 data indicating the variation of the power law exponents $\eta_{0}, \eta_{1}$, and $\eta_{2}$ with density. Due to the lack of extensive statistical averaging, only qualitative trends can be deduced, but it is nevertheless clear that there is in fact little difference between the first three lattice vectors, regardless of the density range, and that the decay exponent $\eta_{0}$ for the first Bragg point will definitely not be very close to the predicted range of $1 / 4-1 / 3$.

We thus see that the results of a more detailed analysis do not really support the initial conjecture regarding pure bond-orientational order in the intermediate region. We have to conclude that a result such as shown in Fig. 1 is apparently much less telltale than expected at the outset. However, this does not necessarily contradict the Murray et al. claim of having successfully identified a hexatic phase in the experimental colloidal sphere system. In fact, if the 

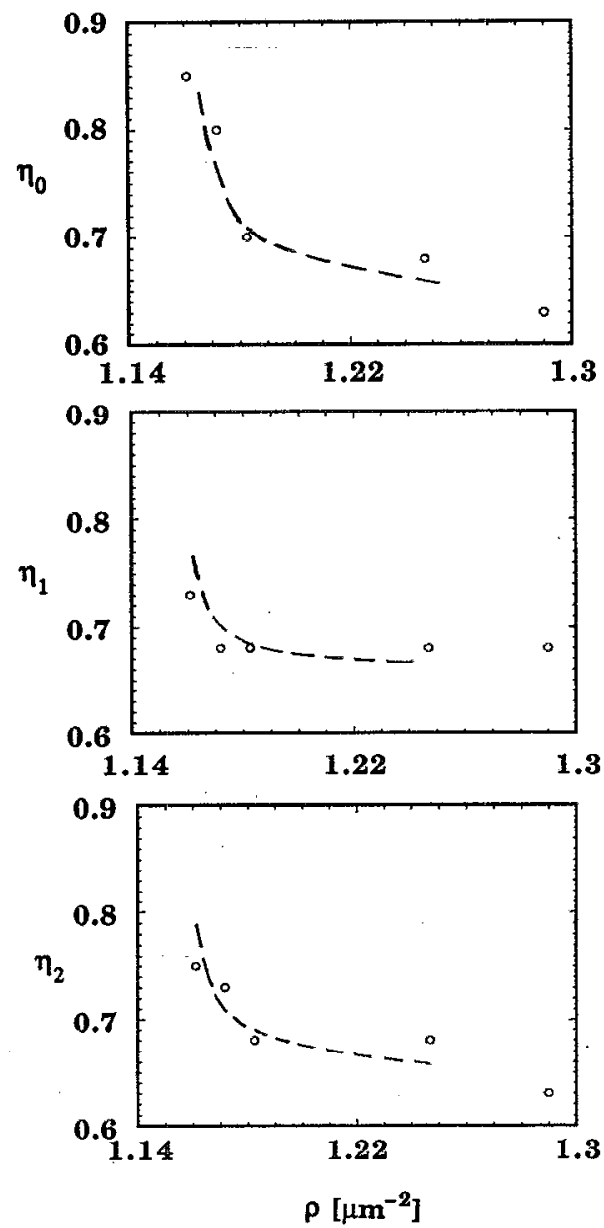

FIG. 4. Power law exponents $\eta_{G}$, as a function of density, obtained by fitting translational correlation functions $g_{G}(r)$ for first three reciprocal lattice vectors to algebraic decay laws. The lines drawn are only guides for the eye.

latter detailed analysis of translational order is applied to some of the colloidal sphere data the predictions of KTHNY theory appear to be reasonably well obeyed, ${ }^{3(d), 11}$ in contrast to what is found here. Obviously there must a significant difference between experiment and simulation, a point to which we will return in Sec. IV.

\section{B. Topological defects}

The dissociation of defect pairs is at the heart of KTHNY theory. Figure 5 shows the evolution of the total defect density (i.e., the fraction of all particles with $\leqslant 5$ and $\geqslant 7$ neighbors) in a 22 million step run at a system density of $\rho=1.170 \mu \mathrm{m}^{-2}$. This is a density in the intermediate region-slightly on the solid side-and the run corresponds to $\approx 8 \mathrm{~s}$ of laboratory time. (We note that the dynamics in our system are not strictly Newtonian because of the use of an algorithm for canonical ensemble sampling.) This is a time range that is not very far from the equilibration time that is implicit in the experimental procedure of Tang et $a .^{5}$ It is also clear that the 22 million time steps exceed the sampling-whether by molecular dynamics or Monte Carlo-in virtually all previous computer

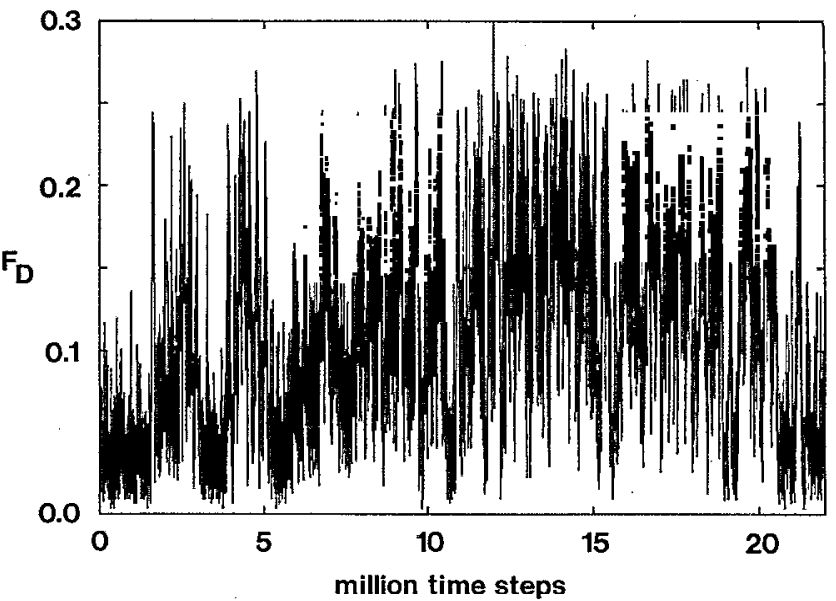

FIG. 5. Fluctuation of defect density $F_{D}$ (=fraction of particles that are not sixfold coordinated in a Voronoi tessellation) during 22 million step run at density $\rho=1.170 \mu \mathrm{m}^{-2}$.

simulations of 2D melting by at least one, if not several orders of magnitude (regardless of the kind of system).

The pattern seen is quite dramatic. The defect density does not only fluctuate between extreme values of $\approx 0 \%$ and $\approx 30 \%$, but one can also infer fluctuation cycles of considerable length, some of them apparently extending over millions of time steps. In addition, up to about 20 million time steps there is an apparent drift towards an overall higher defect density. However, the trend during the last few million time steps make this conclusion less definite, and the pattern seen would also be consistent with the presence of one long oscillation of $\geqslant 30$ million time steps.

We do not have enough data for a detailed analysis, but we can still conclude that correlation times of $\tau \geqslant 10 \mathrm{~s}$ are contributing to the dynamics of our system. The correlation times for translational and orientational motion in the experimental colloidal sphere system (as obtained from analysis of van Hove correlation functions) were found to range between 0.1 and $10 \mathrm{~s}^{3(\mathrm{~b})}$ This seems to be qualitatively consistent with our findings.

Murray puts strong emphasis on the need for sufficient equilibration. ${ }^{11}$ The colloidal sphere experiments were indeed carried out after waiting for up to a few days, rationalized with the suggestion that dislocation climb across a translational correlation length of the system is important for equilibration., ${ }^{3,1}$ Murray has estimated that the time for dislocation climb is about $\approx 10 \mathrm{~h}$ in a system of 0.305 $\mu \mathrm{m}$ sized spheres. ${ }^{11}$ Following this argument, proper equilibration of the Yukawa system in the present simulation setup would require enormous runs, vastly exceeding the length of our 22 million step run. Even if the latter argument should not be valid, we are still left with a drastic illustration of the sampling problem in simulations of 2D phase transitions. It is likely that a comparable problem also affects the experiments of Tang et al. ${ }^{5}$ This would naturally explain the generally great resemblance of their observations with ours. 

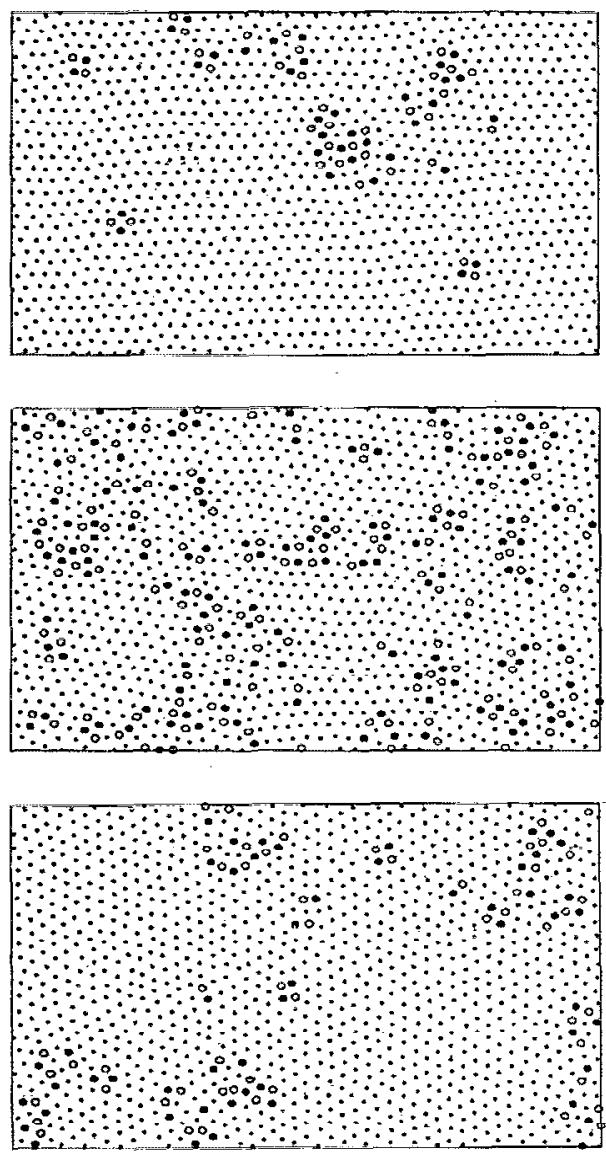

FIG. 6. Defect structure in three representative configurations for density $\rho=1.170 \mu \mathrm{m}^{-2}$. Open and filled circles denote five- and sevenfold coordinated particles, respectively, as determined by Voronoi analysis.

We now turn to the defect structure. Within KTHNY theory, we expect 5-7-5-7 quartets in the crystal, 5-7 pairs in the hexatic, and isolated 5's and isolated 7's in the liquid. Figure 6 displays the defect pattern in three arbitrary configurations of the long run at an intermediate system density. An identifiable fraction of free, or incipiently free, dislocations was observed in the intermediate region of the colloidal suspension experiments ${ }^{3,5}$ and also in simulation studies of the $r^{-12}$ and Coulomb systems. ${ }^{14,19}$ According to the figure, the Yukawa system is no exception to this rule, i.e., occasionally we do find "free" dislocations that are separated by at least a few nearest-neighbor distances. It can also be seen that the defect structure is in general very complex and that clustering of dislocations is the norm, similar to previous observations in the other systems. ${ }^{3,5,14,19}$

Overall, the "clumping" of defects in the pictures looks more similar to what is seen in the colloidal sphere experiments of Tang et al. ${ }^{5}$ than to what is seen by Murray et al. $^{3(\mathrm{c}), 3(\mathrm{~d}), 11,33}$ It is tempting to associate this clumping with lack of equilibration, as previously suggested by Murray. ${ }^{11}$ Still, there is not really a pronounced tendency for the clumping to occur in the form of grain boundaries which are so central to Chui's theory of first-order melting. ${ }^{34}$ There are other differences with respect to the precise defect structure between the various systems, but we have not analyzed these in any detail. We finally did not investigate whether pairs of dislocations dissociate and recombine during the course of the simulation. Such events have not been seen in the experimental system of Tang et al., and this absence has then been used as an argument against the applicability of KTHNY theory. ${ }^{5}$ However, as pointed out by Murray et al. ${ }^{3(\mathrm{~d})}$ the theory is based on a renormalization group treatment and the defect dissociation may take place on an arbitrarily large length scale. Hence, this does not provide a very sensitive test.

\section{CONCLUSIONS}

Does the melting transition in our simulated Yukawa system really follow the scenario of KTHNY theory? Based on the evidence shown we do not believe that the intermediate phase as obtained in this set of simulations can be called a true hexatic. This is so despite the fact that two central expectations of KTHNY theory are easily confirmed, namely a delay between the divergences of translational and orientational order upon melting and the presence of "free" dislocations in the intermediate phase. It is only upon closer inspection that disagreements between theory and simulation become obvious. In this sense, we fully agree with the skepticism of Tang et al. ${ }^{5}$ concerning hasty confirmations of the theoretical predictions, whether in an experimental or in a simulated system.

It may nevertheless be going too far, for more than one reason, to call the agreements between theory and simulation entirely fortuitous. First, indications are that the firstorder and KTHNY transitions are very close, such that one of them barely pre-empts the other. Most likely, the actual melting mechanism-whichever one that may bewill still be strongly influenced by the other. ${ }^{35}$ Second, we cannot exclude the possibility that much more extensive sampling will still give us an intermediate phase that is genuinely hexatic in character. This seems to be a particularly intriguing possibility considering the similarity of our simulation results with the experimental data of Tang et al..$^{5}$ As mentioned earlier, the latter may well suffer from an equilibration problem if compared with the experimental procedure of Murray et al. ${ }^{3}$

Now proceeding to the experimental data, does the melting transition in any of the colloidal sphere systems really follow the scenario of KTHNY theory? Although we have shown that the crucial initial observation ${ }^{3(a)}$ of a delay in the divergence for translational and orientational order is not a strong indicator for the presence of a hexatic phase, we still believe that there are good reasons to answer the question in the affirmative, at least with respect to the data of Murray et al. ${ }^{3}$ The latter have been shown to be in agreement with subtle predictions of the theory, ${ }^{3(d)}$ and at the same time there are no observations that would literally and unequivocally contradict any predictions. The experiments of Tang et al. ${ }^{5}$ on the other hand, appear to pose more of a problem. Since their data resemble so much our simulation data it would not be too surprising if all our reservations concerning the applicability of the theory would equally apply to this set of experiments. 
Thus there is apparently a discrepancy between the data of Murray et $a l^{3}$ on the one hand and the data of Tang et $a l^{5}$ and the present simulation data on the other hand. What is the origin of this discrepancy? We find it helpful to again successively address the points put forth in the Introduction of this paper.

(1) It has occasionally been conjectured that the type of transition may depend on the nature of the potential function, with "soft" interaction potentials favoring a KTHNY-type phase transition since only in the latter type of system short wavelength effects (neglected in the theory) are not expected to be quite that dominant. Examples for a systematic variation in the interaction potential can be given for both experiment and simulation. Thus the two sets of experiments by Murray et al. ${ }^{5}$ and Tang et al. ${ }^{3}$ were carried out with spheres of 0.305 and $1.0 \mu \mathrm{m}$ diam, respectively, while it has been estimated that the screening length $1 / \kappa$ should be comparable in the two cases. ${ }^{3(d)}$ Hence, there is an implicit difference in the range of the interaction potential, with the Tang et al. system being more hardsphere-like. Murray et al. have speculated that this could explain some of the differences in the melting behavior of the two cases. ${ }^{3(d)}$ A similar variation is encountered if we compare the present simulation of a Yukawa (=truncated Coulomb) system with the simulation of the full Coulomb system by Bedanov et al. ${ }^{19}$ Unfortunately, no run times are given for the latter, rather old study and it is difficult to assess its apparently "easy" confirmation of the predictions of KTHNY theory. ${ }^{19}$ Because of the true long-range character of the interactions, sampling in the true Coulomb system is naturally expected to be even more difficult than in the Yukawa system.

A more complex issue is the difference between the interaction potential that is actually effective in the experiment vs the one in the simulation. We note that the width in density of the intermediate region is only $\approx 1 \%$ in the current study of the Yukawa system, as opposed to the about $4 \%-8 \%$ (depending on the identity of the colloid) in the experimental case. ${ }^{3(a), 3(d), 5}$. Many-body potentials may be necessary to realistically model colloidal spheres between glass plates with induced image charges. In this context, we mention that the introduction of explicit polarizability has more recently been one of the major innovations in the routine computer simulation of bulk aqueous systems. Implementation of similar techniques in simulations of $2 \mathrm{D}$ melting would be a most interesting development.

(2) A major issue identified in the present study concerns the amount of sampling. It is the extremely long equilibration time that sets the experiments of Murray et $a l^{3}$ most prominently apart from the simulations carried out here and from the experiments of Tang et $a l^{5}{ }^{5}$ In a simulation study of the $r^{-12}$ system, we already presented some evidence that kinetic bottlenecks are a big impediment for the exploration of configuration space during a 2D phase transition. ${ }^{14}$ It is easily conceivable that only this prevents us from generating a hexatic phase. Of course, our findings also put in doubt virtually all of the numerous other published simulation studies of 2D melting. Much longer runs, possibly using different ensembles and new sampling schemes, are called for. Naturally, there are additional problems with the setup of $2 \mathrm{D}$ computer simulations, such as related to system size ${ }^{27}$ boundary and initial conditions, ${ }^{14}$ substrate effects, and the possibly nonnegligible $3 \mathrm{D}$ character that is left by the experimental confinement.

(3) A final source of discrepancy between experiment and simulation is the data analysis and interpretation. The results presented in Sec. III illustrate vividly how much care has to be exercised to identify evidence for KTHNYtype melting that is only superficial (or even deceptive). We find that there is often little difference between the data from experiment and simulation if these are only analyzed in a uniform manner. ${ }^{14}$

In summary, we suspect that a true hexatic has not been obtained in any simulation study to date, including the present study of the Yukawa system. This is primarily a problem of insufficient sampling. Choice of the "right" potential function might considerably facilitate the search for evidence of KTHNY melting. Even if such evidence has been obtained, careful analysis has to be carried out in order to recognize agreements with theoretical predictions that are only coincidental. Truly penetrating tests have to be devised, such as related to the predicted temperature dependence of the various correlation lengths. Because of simultaneous and unavoidable changes in the screening length of the colloidal interactions such a test cannot easily be carried out experimentally, but can still be accomplished in straightforward computer simulations with a suitable potential function.

\section{ACKNOWLEDGMENTS}

Partial support of this research by a Rackham Faculty Grant (No. 386294) is gratefully acknowledged. The computations were performed with resources provided by a NSF Instrumentation Grant (No. CHE-8917309) to the Department of Chemistry of the University of Michigan.

${ }^{\text {I }}$ R. Pieranski, Contemp. Phys. 24, 25 (1983).

${ }^{2}$ D. Thirumalai, J. Phys. Chem. 93, 5637 (1989).

${ }^{3}$ (a) C. A. Murray and D. H. Van Winkle, Phys. Rev. Lett. 58, 1200 (1987); (b) C. A. Murray and R. A. Wenk, ibid. 62, 1643 (1989); (c) C. A. Murray, D. H. Van Winkle, and R. A. Wenk, Phase Trans. 21, 93 (1990); (d) C. A. Murray, W. O. Sprenger, and R. A. Wenk, Phys. Rev. B 42, 688 (1990).

${ }^{4}$ A. J. Armstrong, R. C. Mockler, and W. J. O'Sullivan, J. Phys. Condensed Matter 1, 1707 (1989).

${ }^{5}$ Y. Tang, A. J. Armstrong, R. C. Mockler, and W. J. O'Sullivan, Phys. Rev. Lett. 62, 2401 (1989); Phase Trans. 21, 75 (1990).

${ }^{\circ}$ K. J. Strandburg, Rev. Mod. Phys. 60, 161 (1988).

${ }^{7}$ Bond Orientational Order in Condensed Matter Systems, edited by K. J. Strandburg (Springer, New York, 1992).

${ }^{8}$ J. M. Kosterlitz and J. D. Thouless, J. Phys. C 6, 1181 (1973).

${ }^{9}$ D. R. Nelson and B. I. Halperin, Phys. Rev. B 19, 2457 (1979); A. P. Young, ibid. 19, 1855 (1979); A Zippelius, B. I. Halperin, and D. R. Nelson, ibid. 22, 2514 (1980).

${ }^{10}$ R. Geer, T. Stoebe, C. C. Huang, R. Pindak, G. Srajer, J. W. Goodby, M. Cheng, J. T. Ho, and S. W. Hui, Phys. Rev. Lett. 66, 1322 (1991); R. Geer, T. Stoebe, C. C. Huang, R. Pindak, J. W. Goodby, M. Cheng, J. T. Ho, and S. W. Hui, Nature 355, 152 (1992).

${ }^{11} \mathrm{C}$. A. Murray, in Ref. 7, p. 137.

${ }^{12}$ J. A. Zollweg and G. V. Chester, Phys. Rev. B 46, 11186 (1992).

${ }^{13}$ J. Lee and K. J. Strandburg, Phys. Rev. B 46, 11190 (1992). 
${ }^{14}$ K. J. Naidoo, J. Schnitker, and J. D. Weeks, Mol. Phys. 80, 1 (1993).

${ }^{15} \mathrm{~K}$. J. Strandburg, in Ref. 7, p. 32.

${ }^{16}$ R. C. Gann, S. Chakravarty, and G. V. Chester, Phys. Rev. B 20, 326 (1979).

${ }^{17}$ R. Morf, Phys. Rev. Lett. 43, 931 (1979).

${ }^{18}$ R. K. Kalia, P. Vashista, and S. W. de Leeuw, Phys. Rev. B.23, 4794 (1981).

${ }^{19}$ V. M. Bedanov, G. V. Gadiyak, and Y. E. Lozovik, Sov. Phys. JETP 61, 967 (1985).

${ }^{20}$ H. Cheng, P. Dutta, D. E. Ellis, and R. Kalia, J. Chem. Phys. 85, 2232 (1986).

${ }^{21}$ (a) D. Hone, S. Alexander, P. M. Chaikin, and P. Pinkus, J. Chem. Phys. 79, 1474 (1983); (b) S. Alexander, P. M. Chaikin, P. Grant, G. J. Morales, P. Pinkus, and D. Hone, ibid. 80, 5776 (1984).

${ }^{22}$ (a) K. Kremer, M. O. Robbins, and G. S. Grest, Phys. Rev. Lett. 57, 2694 (1986); (b) K. Kremer, G. S. Grest, and M. O. Robbins, J. Phys. A 20, L181 (1987); (c) M. O. Robbins, K. Kremer, and G. S. Grest, J. Chem. Phys. 88, 3286 (1988).

${ }^{23}$ R. O. Rosenberg and D. Thirumulai, Phys. Rev. A 36, 5690 (1987).
${ }^{24}$ E. J. Meijer and D. Frenkel, J. Chem. Phys. 94, 2269 (1991).

${ }^{25}$ J. C. Zahorchak, R. Kesavamoorthy, R. D. Coalson, and S. A. Asher, J. Chem. Phys. 96, 6873 (1992):

${ }^{26}$ M. J. Stevens and M. O. Robbins, J. Chem. Phys. 98, 2319 (1993).

${ }^{27}$ S. Toxvaerd, Phys. Rev. Lett. 51, 1971 (1983).

${ }^{28}$ J. A. Zollweg, G. V. Chester, and P. W. Leung, Phys. Rev. B 39, 9518 (1989).

${ }^{29}$ C. A. Murray, W. O. Sprenger, and R. A. Wenk, J. Phys. Condensed Matter 2, SA385 (1990).

${ }^{30}$ H. Löwen, J.-P. Hansen, and P. A. Madden, J. Chem. Phys. 98, 3275 (1993).

${ }^{31}$ M. P. Allen and D. Tildesley, Computer Simulation of Liquids (Clarendon, Oxford, 1987).

${ }^{32}$ J. D. Weeks, Phys. Rev. B 24, 1530 (1981).

${ }^{33}$ Note that the defect patterns in Ref. 3(a) are incorrect because of a faulty Voronoi construction. The defect patterns in Refs. 3(c), 3(d), and 11 are correct.

${ }^{34}$ S. T. Chui, Phys. Rev. B 28, 178 (1983).

${ }^{35}$ C. Udink and D. Frenkel, Phys. Rev. B 35, 6933 (1987). 